\title{
The Picard group of a noncommutative algebraic torus
}

\author{
Yuri Berest, Ajay Ramadoss, and Xiang Tang
}

\begin{abstract}
Let $A_{q}:=\mathbb{C}\left\langle x^{ \pm 1}, y^{ \pm 1}\right\rangle /(x y-q y x)$. Assuming that $q$ is not a root of unity, we compute the Picard group $\operatorname{Pic}\left(A_{q}\right)$ of the algebra $A_{q}$, describe its action on the space $\mathcal{R}\left(A_{q}\right)$ of isomorphism classes of rank 1 projective modules and classify the algebras Morita equivalent to $A_{q}$. Our computations are based on a 'quantum' version of the Calogero-Moser correspondence relating projective $A_{q}$-modules to irreducible representations of the double affine Hecke algebras $\mathbb{W}_{t, q^{-1 / 2}}\left(S_{n}\right)$ at $t=1$. We show that, under this correspondence, the action of $\operatorname{Pic}\left(A_{q}\right)$ on $\mathcal{R}\left(A_{q}\right)$ agrees with the action of $\operatorname{SL}_{2}(\mathbb{Z})$ on $\mathbb{T}_{t, q^{-1 / 2}}\left(S_{n}\right)$ constructed by Cherednik [C1], [C2]. We compare our results with the smooth and analytic cases. In particular, when $|q| \neq 1$, we find that $\operatorname{Pic}\left(A_{q}\right) \cong \operatorname{Auteq}\left(\mathcal{D}^{\mathrm{b}}(X)\right) / \mathbb{Z}$, where $\mathcal{D}^{\mathrm{b}}(X)$ is the bounded derived category of coherent sheaves on the elliptic curve $X=\mathbb{C}^{*} / \mathbb{Z}$.
\end{abstract}

Mathematics Subject Classification (2010). 16S35; 16D40, 16W20, 16S80.

Keywords. Noncommutative algebraic torus, quantum Weyl algebra, projective module, Morita equivalence, Picard group, double affine Hecke algebra.

\section{Introduction}

Let $\mathbb{C}\left\langle x^{ \pm 1}, y^{ \pm 1}\right\rangle$ be the group algebra of the free group on two generators $x$ and $y$, with coefficients in $\mathbb{C}$. We define $A_{q}:=\mathbb{C}\left\langle x^{ \pm 1}, y^{ \pm 1}\right\rangle /(x y-q y x)$ for a fixed parameter $q \in \mathbb{C}$. Unless specified otherwise, we will assume in this paper that

$$
q^{n} \neq 1 \quad \text { for all } n \in \mathbb{N} \text {. }
$$

Under this condition, we will (a) classify finitely generated (right) projective modules of $A_{q}$, (b) compute the Picard group $\operatorname{Pic}\left(A_{q}\right)$ and describe its action on projective modules, and (c) classify the algebras Morita equivalent to $A_{q}$.

There are several reasons to clarify these questions. First, $A_{q}$ may be thought of as a ring of functions on a noncommutative algebraic torus. Now, for a noncommutative smooth torus $\mathscr{A}_{q}$, the answers to (a), (b) and (c) are well known and well understood (mostly thanks to the work of Rieffel, see [R1], [R2], [R3], [K1]). Geometrically, the algebras $\left\{\mathscr{A}_{q}\right\}$ arise as deformations of the ring $\mathcal{C}^{\infty}(\mathbb{T})$ of smooth functions on the two-dimensional torus $\mathbb{T}=\mathbb{S}^{1} \times \mathbb{S}^{1}$, and as such, these are fundamental examples of noncommutative differentiable manifolds in the sense of A. Connes [Co]. On the 
other hand, algebraically, $\mathcal{A}_{q}$ is just a certain completion of $A_{q}$, and it is natural to ask how the projective modules, Picard groups, Morita equivalences, etc. behave under this completion. One might expect that $A_{q}$ is 'too rigid' compared to $\mathcal{A}_{q}$, and the answers to the above questions are trivial. We will demonstrate that this is not the case, and although the properties of $A_{q}$ and $\mathcal{A}_{q}$ are indeed very different, the answers to (a), (b) and (c) in the algebraic case are at least as meaningful and interesting as in the smooth case.

Second, $A_{q}$ may be viewed as a 'quantum' (or multiplicative) Weyl algebra. Under the assumption (1), the ring-theoretic properties of $A_{q}$ are indeed similar to those of the usual Weyl algebra $A_{1}=\mathbb{C}\langle x, y\rangle /(x y-y x-1)$ (see [J]). From this perspective, our answer to $(a)$ should not be very surprising. It is known that the rank one projective modules of $A_{1}$ are isomorphic to ideals and as such, can be parametrized by certain smooth algebraic varieties $C_{n}$ called the Calogero-Moser spaces (see [BW1], [BW2], [BC]). Similarly, the ideals of $A_{q}$ are described by a certain 'quantum' version of the Calogero-Moser spaces $\ell_{n}^{q}$ (see Theorem 1), and, in fact, a similar geometric description exists for more general classes of noncommutative algebras (see, e.g., [KKO], [NB], [NS]). What is surprising is the fact that the classification of ideals of $A_{q}$ allows one to compute the Picard group $\operatorname{Pic}\left(A_{q}\right)$ of the category of all $A_{q}$-modules. By contrast, the Picard group Pic $\left(A_{1}\right)$ of the Weyl algebra was computed by Stafford who showed that $\operatorname{Pic}\left(A_{1}\right)=\operatorname{Aut}\left(A_{1}\right)$, using reduction to fields of characteristic $p>0$ (see [St]). A different proof of Stafford's result can be found in [CH]: it relies on Dixmier's classification [D] of maximal commutative subalgebras of $A_{1}$. Both proofs are fairly involved and indirect; in particular, they do not follow from the results of [BW1], [BW2], [BC]. To the best of our knowledge, the group $\operatorname{Pic}\left(A_{q}\right)$ has not appeared in the literature: we therefore consider its computation (Theorem 3 ) together with a related Morita classification (Theorem 5) as the main results of this paper.

Third, the quantum Calogero-Moser spaces $\mathcal{C}_{n}^{q}$ parametrizing the ideals of $A_{q}$ can be defined as the spectra of spherical subalgebras of Cherednik's double affine Hecke algebra (DAHA) $\mathbb{U}_{1, q^{-1 / 2}}\left(S_{n}\right)$ (see [O]). Our results then imply that there is a natural bijection between the set of equivalence classes of irreducible representations of $\mathbb{Q}_{1, q^{-1 / 2}}\left(S_{n}\right)$ amalgamated for all $n$ and the set $\mathcal{R}\left(A_{q}\right)$ of isomorphism classes of ideals of $A_{q}$. In the rational case, the analogous bijection was first discovered in [EG], and its proof was given in [BCE]. In this paper, we will extend the construction of $[\mathrm{BCE}]$ to the quantum case (see Section 5). A new interesting observation is that, under the Calogero-Moser correspondence, the action of $\operatorname{Pic}\left(A_{q}\right)$ on $\mathcal{R}\left(A_{q}\right)$ corresponds to the action of $\mathrm{SL}_{2}(\mathbb{Z})$ on $\mathbb{U}_{1, q^{-1 / 2}}\left(S_{n}\right)$ constructed (and exploited in many applications) by Cherednik [C1], [C2].

Finally, $A_{q}$ is a fundamental noncommutative algebra which plays a role in many areas of mathematics and physics. As a specific motivation to clarify questions (a), (b) and (c) for $A_{q}$, we mention a recent appearance of this algebra in knot theory: it is shown in [FGL] (see also [G]) that the classical invariants of knots - the so-called $A$-polynomials - can be naturally quantized, and the corresponding quantizations are given by certain noncyclic ideals of $A_{q}$. Comparing these ideals for different knots 
is not an easy problem. Having a general classification and canonical forms for all ideals of $A_{q}$ should be helpful in this context.

As another application, we should mention the link to integrable systems. The quantum Calogero-Moser spaces $\ell_{n}^{q}$ first appeared in [FR] in connection with 2D Toda hierarchy and the Ruijsenaars-Schneider system; since then they were discussed in numerous papers on integrable systems. By analogy with the Weyl algebra (see [W1], [BW1]), the ideals of $A_{q}$ are related to algebraic solutions of these systems, which in turn can be described in terms of $q$-version of the adelic Grassmannian of G. Wilson [W1], [W2] (see [CH1], [CN] and [BC1] for more details).

The paper is organized as follows. In Section 2, we define the Calogero-Moser spaces $\ell_{n}^{q}$ and give our classification of ideals of $A_{q}$. In Section 3, we compute the Picard group $\operatorname{Pic}\left(A_{q}\right)$ and discuss some implications. In Section 4, we describe the action of $\operatorname{Pic}\left(A_{q}\right)$ on the Calogero-Moser spaces and classify the algebras Morita equivalent to $A_{q}$ in terms of this action. In Section 5, we explain the relation between the DAHA and $A_{q}$ and outline proofs of our main results. Finally, in Section 6, we compare the properties of $A_{q}$ with the properties of noncommutative smooth tori $\mathcal{A}_{q}$.

Acknowledgements. We are very grateful to O. Chalykh for his remarks and suggestions; although he is formally not a coauthor, many ideas in this paper belong to him (in particular, Theorem 1 in its current form first appeared in [BC1], where it was proven by elementary methods). We are also grateful to M. Rieffel for his encouragement to write this paper and for providing us with references [K1], [K2] and [Wa]. The first author would like to thank J. Alev, A. Polishchuk and G. Wilson for interesting discussions and comments.

The work of Yu. B. and X. T. was partially supported by NSF grants DMS 0901570 and DMS 0900985, respectively. A. R. is currently funded by the Swiss National Science Foundation (Ambizione Beitrag Nr. PZ00P2-127427/1).

\section{The Calogero-Moser correspondence for $A_{q}$}

Under the assumption (1), the algebra $A_{q}$ is a simple Noetherian domain of homological dimension 1 (see $[\mathrm{J}]$, Theorem 2.1). In that case, it is known that every right projective module of rank $\geq 2$ is free, while every rank one projective module is isomorphic to a right ideal of $A_{q}$ (see [We], Theorem 1). Thus the problem of classifying projectives over $A_{q}$ reduces to classifying the right ideals of $A_{q}$. Below, we will give an explicit classification of such ideals similar to the classification of ideals in the Weyl algebra $A_{1}$.

For an integer $n \geq 1$, let $\tilde{e}_{n}^{q}$ denote the space of matrices

$$
\left\{(X, Y, i, j) \mid X, Y \in \mathrm{GL}_{n}(\mathbb{C}), i \in \mathbb{C}^{n}, j \in \operatorname{Hom}\left(\mathbb{C}^{n}, \mathbb{C}\right)\right\},
$$

satisfying the equation

$$
q X Y-Y X+i j=0 .
$$


The group $\mathrm{GL}_{n}(\mathbb{C})$ acts on $\tilde{\mathcal{C}}_{n}^{q}$ in the natural way:

$$
(X, Y, i, j) \mapsto\left(g X g^{-1}, g Y g^{-1}, g i, j g^{-1}\right), \quad g \in \mathrm{GL}_{n}(\mathbb{C}),
$$

and this action is free for all $n$. We define the $n$-th Calogero-Moser space $\ell_{n}^{q}$ to be the quotient variety $\widetilde{C}_{n}^{q} / \mathrm{GL}_{n}(\mathbb{C})$ modulo (2). $\mathcal{C}_{n}^{q}$ are smooth irreducible affine symplectic varieties of (complex) dimension $2 n$ (see [O]). We set $\ell^{q}:=\bigsqcup_{n \geq 0} \ell_{n}^{q}$, assuming that $\ell_{0}^{q}$ is a point.

In a slightly more invariant way, we may think of $\ell^{q}$ as the space of (isomorphism classes of triples $(V, X, Y)$, where $V$ is a finite-dimensional complex vector space, and $X, Y$ are automorphisms of $V$ satisfying the condition

$$
\operatorname{rk}\left(q X Y X^{-1} Y^{-1}-1_{V}\right)=1 .
$$

Now, for each $n \geq 0$, there is a natural action on $\ell_{n}^{q}$ by the lattice $\mathbb{Z}^{2}$ :

$$
(V, X, Y) \mapsto\left(V, q^{k} X, q^{m} Y\right), \quad(k, m) \in \mathbb{Z}^{2} .
$$

We write $\bar{\ell}_{n}^{q}:=\ell_{n}^{q} / \mathbb{Z}^{2}$ for the corresponding quotient spaces, and set $\bar{\ell}^{q}:=$ $\bigsqcup_{n>0} \bar{\varphi}_{n}^{q}$.

Let $\mathcal{R}^{q}:=\mathcal{R}\left(A_{q}\right)$ be the set of isomorphism classes of right ideals of $A_{q}$. Our first main result is the following

Theorem 1. There is a natural bijection $\omega: \bar{e}^{q} \stackrel{\sim}{\longrightarrow} \mathcal{R}^{q}$.

As in the Weyl algebra case, the bijection $\omega$ can be described quite explicitly; it is induced by the map $\mathcal{C}^{q} \rightarrow \mathcal{R}^{q}$, assigning to $(V, X, Y) \in \mathcal{C}^{q}$ the class of (isomorphic) fractional ideals

$$
\begin{aligned}
& M_{x}=\operatorname{det}\left(X-x 1_{V}\right) \cdot A_{q}+\kappa^{-1} \operatorname{det}\left(Y-y 1_{V}\right) \cdot A_{q}, \\
& M_{y}=\operatorname{det}\left(Y-y 1_{V}\right) \cdot A_{q}+\kappa \operatorname{det}\left(X-x 1_{V}\right) \cdot A_{q},
\end{aligned}
$$

where $\kappa$ and $\kappa^{-1}$ are given by the following elements in the quotient field of $A_{q}$ :

$$
\begin{aligned}
\kappa & =1+j\left(q Y-y 1_{V}\right)^{-1}\left(X-x 1_{V}\right)^{-1} i, \\
\kappa^{-1} & =1-j\left(X-q x 1_{V}\right)^{-1}\left(Y-y 1_{V}\right)^{-1} i .
\end{aligned}
$$

Theorem 1 thus implies that the right ideals of $A_{q}$ (and hence rank one projective $A_{q}$-modules) are classified by the conjugacy classes of pairs of matrices $(X, Y) \in$ $\mathrm{GL}(V) \times \mathrm{GL}(V)$ satisfying (3). Furthermore, every ideal of $A_{q}$ is isomorphic to one of the form (4)-(5), with pairs $(X, Y)$ and $\left(X^{\prime}, Y^{\prime}\right)$ corresponding to isomorphic ideals if and only if $\left(X^{\prime}, Y^{\prime}\right) \sim\left(q^{k} X, q^{m} Y\right)$ for some $k, m \in \mathbb{Z}$. As mentioned in the Introduction, this result is similar to that for the Weyl algebra $A_{1}$, and in the existing literature there are several different proofs (see [BW1], [BW2], [BC], [BCE]). Each of these proofs can be extended to the quantum case. In Section 5, we will outline a proof generalizing the arguments of $[\mathrm{BC}],[\mathrm{BCE}]$ : the advantage of this approach is that it exhibits an interesting connection between $A_{q}$ and the representation theory of double affine Hecke algebras $\mathbb{U}_{1, q^{-1 / 2}}\left(S_{n}\right)$, which may be of independent interest.

We now discuss some interesting implications of Theorem 1. 


\section{The Picard group of $A_{q}$}

We begin by introducing notation. Being a Noetherian domain, the algebra $A_{q}$ can be embedded into a quotient skew-field, which we denote by $Q$. The spaces of nonzero (Laurent) polynomials in $x$ and $y$ form two Ore subsets in $A_{q}$ : we write $\mathbb{C}(x)\left[y^{ \pm 1}\right] \subset Q$, resp. $\mathbb{C}(y)\left[x^{ \pm 1}\right] \subset Q$, for the corresponding localizations. Every element $a \in \mathbb{C}(x)\left[y^{ \pm 1}\right]$ can be uniquely written in the form $a=\sum_{m \leq i \leq n} a_{i}(x) y^{i}$, with $a_{i} \in \mathbb{C}(x)$ and $a_{m}, a_{n} \neq 0$. We call $a_{n}(x)$ the leading coefficient of $a$, and the difference $n-m$ the degree of $a$.

Lemma 1. Every ideal $M$ in $A_{q}$ is isomorphic to a fractional ideal $M_{x}$ satisfying

(1) $M_{x} \subset \mathbb{C}(x)\left[y^{ \pm 1}\right]$ and $M_{x} \cap \mathbb{C}\left[x^{ \pm 1}\right] \neq\{0\}$;

(2) the leading coefficients of all elements in $M_{x}$ belong to $\mathbb{C}\left[x^{ \pm 1}\right]$;

(3) $M_{x}$ contains an element with a constant leading coefficient.

If $M_{x}$ and $M_{x}^{\prime}$ are two fractional ideals of $A_{q}$, both isomorphic to $M$, and satisfying (1)-(3), then there is a unit $u \in A_{q}$ such that $M_{x}^{\prime}=u M_{x}$.

Proof. The proof is essentially the same as in the Weyl algebra case (see [BW2], Lemma 5.1). First, by [St], Lemma 4.2, every ideal class of $A_{q}$ contains a representative $M$ such that $M \cap \mathbb{C}\left[x^{ \pm 1}\right] \neq\{0\}$. The leading coefficients of all the elements of $M$ form an ideal in $\mathbb{C}\left[x^{ \pm 1}\right]$; taking a generator $p(x) \in \mathbb{C}\left[x^{ \pm 1}\right]$ of this ideal, we set $M_{x}:=p^{-1} M$. It is easy to see that $M_{x}$ thus defined satisfies the properties (1)-(3).

Now, if $M_{x}^{\prime}$ is another (fractional) ideal isomorphic to $M$, we have $M_{x}^{\prime}=\gamma M_{x}$ for some $\gamma \in Q$. If both $M_{x}$ and $M_{x}^{\prime}$ satisfy (1), then $\gamma$ must be a unit in $\mathbb{C}(x)\left[y^{ \pm 1}\right]$ and hence has the form $\gamma=f(x) y^{k}$, with $f(x) \in \mathbb{C}(x) \backslash\{0\}$ and $k \in \mathbb{Z}$. Property (2) forces $f(x)$ to be polynomial, i. e. $f(x) \in \mathbb{C}\left[x^{ \pm 1}\right]$, and then (3) implies that $f(x)=\alpha x^{m}$ for some $\alpha \in \mathbb{C}^{*}$ and $m \in \mathbb{Z}$. Thus $\gamma=\alpha x^{m} y^{k}$ is a unit in $A_{q}$.

Given any (fractional) ideal $M \subset Q$ of $A_{q}$, its endomorphism ring is naturally identified with a subring of $Q$ :

$$
\operatorname{End}_{A}(M)=\{\gamma \in Q \mid \gamma M \subseteq M\} .
$$

This yields a group embedding $\operatorname{Aut}_{A}(M) \hookrightarrow Q^{*}$, whose image we denote by $U(M)$. Thus $U(M)=\left\{\gamma \in Q^{*} \mid \gamma M=M\right\}$. In particular, if $M=A_{q}$, we get the group of units $U\left(A_{q}\right)$ of $A_{q}$ canonically embedded in $Q^{*}$.

Lemma 2. Let $M_{x}$ be a fractional ideal of $A_{q}$ satisfying the properties (1)-(3) of Lemma 1. Then $U\left(M_{x}\right) \subseteq U\left(A_{q}\right)$. Moreover, if $M_{x}^{\prime} \cong M_{x}$ is another representative satisfying (1)-(3), we have $U\left(M_{x}^{\prime}\right)=U\left(M_{x}\right)$ in $U\left(A_{q}\right)$. Thus the subgroup $U\left(M_{x}\right) \subseteq U\left(A_{q}\right)$ depends only on the class of $M_{x}$ in $\mathcal{R}^{q}$. 
Proof. The fact that $U\left(M_{x}\right) \subseteq U\left(A_{q}\right)$ is immediate from (the last statement of) Lemma 1. It implies that every element in $U\left(M_{x}\right)$ has the form $\gamma=\beta x^{k} y^{m}$, where $\beta \in \mathbb{C}^{*}, k, m \in \mathbb{Z}$. Using the commutation relations of $A_{q}$, it is easy to see that $u \gamma u^{-1}=\tilde{\beta} x^{k} y^{m}$ for any $u \in U\left(A_{q}\right)$. Hence $u U\left(M_{x}\right) u^{-1}=U\left(M_{x}\right)$, which proves the second claim of the lemma.

Remark. Reversing the roles of $x$ and $y$ in the above lemmas, we obtain another set of distinguished representatives $M_{y} \subset \mathbb{C}(y)\left[x^{ \pm 1}\right]$ for any given class in $\mathcal{R}^{q}$. The ideals (4) and (5) appearing in Theorem 1 are examples of such representatives.

The next result is an important consequence of Theorem 1.

Theorem 2. Let $M$ be a noncyclic right ideal of $A_{q}$. Then $\operatorname{Aut}_{A}(M) \cong \mathbb{C}^{*}$.

Proof. First of all, note that $\mathbb{C}^{*} \subseteq U(M) \subset Q$ for any ideal $M$. Now suppose that $\operatorname{Aut}_{A}(M) \nRightarrow \mathbb{C}^{*}$. Choose a representative $M_{x}$ in the isomorphism class of $M$ satisfying the conditions (1)-(3) of Lemma 1 . Since $\operatorname{Aut}_{A}\left(M_{x}\right) \cong \operatorname{Aut}_{A}(M) \nsubseteq \mathbb{C}^{*}$, we have $\mathbb{C}^{*} \subsetneq U\left(M_{x}\right)$.

By Theorem 1, we may assume that $M_{x}$ has the form (4) and take $M_{y}$ to be the second representative (5). The ideals $M_{x}$ and $M_{y}$ are related by $M_{y}=\kappa M_{x}$, where $\kappa=\kappa(x, y) \in Q$ is defined in (6). It follows that

$$
\gamma \in U\left(M_{x}\right) \Longleftrightarrow \kappa \gamma \kappa^{-1} \in U\left(M_{y}\right) .
$$

Now choose $\gamma \in U\left(M_{x}\right) \backslash \mathbb{C}^{*}$. Without loss of generality, we can write $\gamma=x^{k} y^{l}$ for some $(k, l) \neq(0,0)$. Then $\kappa \gamma \kappa^{-1}=\alpha x^{c} y^{d}$ for some $(c, d) \neq(0,0)$ and $\alpha \in \mathbb{C}^{*}$. Whence $\kappa \gamma^{m} \kappa^{-1}=\left(\alpha x^{c} y^{d}\right)^{m}$ for every $m \in \mathbb{Z}$. It follows that, for any $m \in \mathbb{Z}$, there exists $\alpha_{m} \in \mathbb{C}^{*}$ such that

$$
\gamma^{-m} \kappa \gamma^{m} \kappa^{-1}=\alpha_{m} x^{m(c-k)} y^{m(d-l)} .
$$

If $(k, l) \neq(c, d)$, we may choose $m$ so that $\max \{m(c-k), m(d-l)\}>0$. For such $m$, the equation (7) clearly does not hold. Therefore we have $(c, d)=(k, l)$. Further, by comparing the constant terms of the Laurent series expansions on both sides of (7) (for $m=1$ ), we find that $\alpha_{1}=1$. Using the commutation relations in $Q$, we may therefore rewrite the equation (7) for $m=1$ in the form

$$
\kappa(x, y)=\kappa\left(q^{l} x, q^{-k} y\right) .
$$

Expanding now $\kappa$ into the Laurent series

$$
\kappa(x, y)=1+\sum_{s, r \geq 0} a_{s r} y^{-s-1} x^{-r-1}, \quad a_{s r}=q^{s} j Y^{s} X^{r} i,
$$

and substituting it into (8), we get

$$
a_{s r}\left(1-q^{k(s+1)-l(r+1)}\right)=0 \quad \text { for all } r, s \in \mathbb{Z}_{+} .
$$


It follows that $a_{r s}=0$ for all $r, s$ such that $k(s+1) \neq l(r+1)$. Now, since $X \in \mathrm{GL}_{n}(\mathbb{C})$, the characteristic polynomial of $X$ has a nonzero constant term. Hence $1=\sum_{p=1}^{n} c_{p} X^{p}$ for some $c_{p} \in \mathbb{C}$. Suppose that $s, r \in \mathbb{Z}_{+}$satisfy $k(s+1)=$ $l(r+1)$. Then $k$ and $l$ are both nonzero. Since $k(s+1) \neq l(r+p+1)$ for any $p>0$, we have

$$
a_{s r}=q^{s} j Y^{s} X^{r} i=\sum_{p=1}^{n} c_{p} q^{s} j Y^{s} X^{r+p_{i}}=\sum_{p=1}^{n} c_{p} a_{s r+p}=0 .
$$

This shows that $a_{s r}=0$ for all $s, r \in \mathbb{Z}_{+}$. Thus $\kappa=1$ and $M_{x}=M_{y}=A_{q}$. It follows that $M \cong M_{x}$ is a cyclic ideal.

Now, using Theorem 1, we compute the Picard group of the algebra $A_{q}$. Recall that the elements of $\operatorname{Pic}\left(A_{q}\right)$ are the isomorphism classes of invertible bimodules of $A_{q}$, which are symmetric over $\mathbb{C}$. There is a natural group homomorphism $\Omega: \operatorname{Aut}\left(A_{q}\right) \rightarrow \operatorname{Pic}\left(A_{q}\right)$, taking $\sigma \in \operatorname{Aut}\left(A_{q}\right)$ to the class of the bimodule $\left(A_{q}\right)^{\sigma}$, which is isomorphic to $A_{q}$ as a right module, with left action of $A_{q}$ twisted by $\sigma^{-1}$. By [F], Theorem 1, the kernel of this homomorphism is precisely the group $\operatorname{Inn}\left(A_{q}\right)$ of inner automorphisms of $A_{q}$, while $\operatorname{Im}(\Omega)$ consists of those invertible $A_{q}$-bimodules that are cyclic as right modules. Since $A_{q}$ is a domain, an invertible bimodule over $A_{q}$ is just a right ideal $M$ of $A_{q}$ such that $\operatorname{End}_{A_{q}}(M) \cong A_{q}$ as $\mathbb{C}$-algebras. This last condition implies that $\operatorname{Aut}_{A_{q}}(M) \cong U\left(A_{q}\right)$, so by Theorem 2, $M$ is indeed cyclic. Thus $\Omega$ is surjective, and we have

Proposition 1. The canonical sequence of groups

$$
1 \rightarrow \operatorname{Inn}\left(A_{q}\right) \rightarrow \operatorname{Aut}\left(A_{q}\right) \stackrel{\Omega}{\longrightarrow} \operatorname{Pic}\left(A_{q}\right) \rightarrow 1
$$

is exact.

With Proposition 1, the problem of computing $\operatorname{Pic}\left(A_{q}\right)$ reduces to describing the automorphisms of $A_{q}$. Let $\left(\mathbb{C}^{*}\right)^{2}$ denote the direct product of multiplicative groups of $\mathbb{C}$. We define an action of $\mathrm{SL}_{2}(\mathbb{Z})$ on $\left(\mathbb{C}^{*}\right)^{2}$ by

$$
g:(\alpha, \beta) \mapsto\left(\alpha^{a} \beta^{b}, \alpha^{c} \beta^{d}\right), \quad g=\left(\begin{array}{ll}
a & b \\
c & d
\end{array}\right) \in \mathrm{SL}_{2}(\mathbb{Z}),
$$

and form the semidirect product $\left(\mathbb{C}^{*}\right)^{2} \rtimes \mathrm{SL}_{2}(\mathbb{Z})$ relative to this action. The following result is probably well known (see, e.g., [AD1], [AD2]).

Lemma 3. $\operatorname{Aut}\left(A_{q}\right) \cong\left(\mathbb{C}^{*}\right)^{2} \rtimes \mathrm{SL}_{2}(\mathbb{Z})$.

Proof. We first recall the well-known presentation of $\mathrm{SL}_{2}(\mathbb{Z})$ as a quotient of the braid group $B_{2}$ :

$$
\mathrm{SL}_{2}(\mathbb{Z})=\left\langle g_{1}, g_{2} \mid g_{1} g_{2} g_{1}=g_{2} g_{1} g_{2},\left(g_{1} g_{2}\right)^{6}=1\right\rangle .
$$


The braid generators of $g_{1}$ and $g_{2}$ correspond under (10) to the matrices

$$
g_{1}=\left(\begin{array}{ll}
1 & 1 \\
0 & 1
\end{array}\right), \quad g_{2}=\left(\begin{array}{cc}
1 & 0 \\
-1 & 1
\end{array}\right) .
$$

Now, using this presentation, it is easy to check that

$$
g_{1}:(x, y) \mapsto(y x, y), \quad g_{2}:(x, y) \mapsto\left(x, y x^{-1}\right) .
$$

extends to a well-defined group homomorphism $\mathrm{SL}_{2}(\mathbb{Z}) \rightarrow \operatorname{Aut}\left(A_{q}\right)$ of the form

$$
g:(x, y) \mapsto\left(\alpha_{g} y^{b} x^{a}, \beta_{g} y^{d} x^{c}\right),
$$

where $g \in \mathrm{SL}_{2}(\mathbb{Z})$ as in (9) and $\alpha_{g}, \beta_{g} \in \mathbb{C}^{*}$ are some constants depending on $g$. On the other hand, there is an obvious homomorphism $\left(\mathbb{C}^{*}\right)^{2} \rightarrow \operatorname{Aut}\left(A_{q}\right)$, mapping $(x, y) \mapsto(\alpha x, \beta y)$ for $(\alpha, \beta) \in\left(\mathbb{C}^{*}\right)^{2}$. These two homomorphisms fit together giving

$$
\left(\mathbb{C}^{*}\right)^{2} \rtimes \mathrm{SL}_{2}(\mathbb{Z}) \rightarrow \operatorname{Aut}\left(A_{q}\right),
$$

which is easily seen to be injective.

On the other hand, any element $\sigma \in \operatorname{Aut}\left(A_{q}\right)$ takes units to units: in particular, it maps the generators $(x, y)$ to elements of the form $\left(\alpha y^{b} x^{a}, \beta y^{d} x^{c}\right)$, with $\alpha, \beta \in c^{*}$ and $a, b, c, d \in \mathbb{Z}$. The invertibility of $\sigma$ means that $a d-b c= \pm 1$ and the relation $\sigma\left(x y x^{-1} y^{-1}\right)=q$ ensures that $a d-b c=1$. This proves that (11) is surjective.

Under the identification of Lemma 3, the inner automorphisms of $A_{q}$ correspond to the elements $\left(q^{n} x, q^{m} y ; 1\right) \in\left(\mathbb{C}^{*}\right)^{2} \rtimes \mathrm{SL}_{2}(\mathbb{Z})$, with $n, m \in \mathbb{Z}$. Indeed, one can compute easily that the canonical projection $U\left(A_{q}\right) \rightarrow \operatorname{Inn}\left(A_{q}\right), u \mapsto \operatorname{Ad}(u)$, is given on generators by $\operatorname{Ad}(u):(x, y) \mapsto\left(q^{-b} x, q^{a} y\right)$, where $u=\alpha x^{a} y^{b} \in U\left(A_{q}\right)$. Thus, we arrive at the following theorem, which is the main result of this paper.

Theorem 3. With identification of Lemma 3, the canonical map $\Omega$ induces an isomorphism of groups

$$
\operatorname{Pic}\left(A_{q}\right) \cong\left(\mathbb{C}^{*} / \mathbb{Z}\right)^{2} \rtimes \mathrm{SL}_{2}(\mathbb{Z}),
$$

where $\mathbb{Z}$ is identified with the cyclic subgroup of $\mathbb{C}^{*}$ generated by $q$.

We end this section with a side observation. Assume that $|q| \neq 1$. Regarding $\mathbb{C}^{*}$ as complex analytic space, we can then identify the quotient $\mathbb{C}^{*} / \mathbb{Z}$ with a (smooth) elliptic curve $X$. Let $\mathcal{D}^{\mathrm{b}}(X)$ denote the bounded derived category of coherent sheaves on $X$, and let Auteq $\mathcal{D}^{\mathrm{b}}(X)$ be the group of (exact) auto-equivalences of this category. Comparing our Theorem 3 with results of [Or], [ST], we get

Corollary 1. There is a natural group isomorphism

$$
\gamma: \operatorname{Pic}\left(A_{q}\right) \stackrel{\sim}{\longrightarrow} \operatorname{Auteq}\left(\mathcal{D}^{\mathrm{b}}(X)\right) / \mathbb{Z},
$$

where $\mathbb{Z} \subset$ Auteq $\left(\mathcal{D}^{\mathrm{b}}(X)\right)$ corresponds to the subgroup of translation functors on $\mathcal{D}^{\mathrm{b}}(X)$. 
We will briefly explain how to construct the isomorphism $\gamma$. Let $x_{0} \in X$ be the point corresponding to the image of $1 \in \mathbb{C}^{*}$ under the canonical projection $\mathbb{C}^{*} \rightarrow X$. By $[\mathrm{Or}]$ (see also [ST]), the group Auteq $\mathcal{D}^{\mathrm{b}}(X)$ is then generated by the functors

$$
T_{\mathcal{O}}, T_{\mathcal{O}_{x_{0}}} \text { and } R_{x}, L_{x} \quad(x \in X),
$$

where $L_{x}$ is induced by tensoring with the line bundle $\mathcal{O}\left(x-x_{0}\right), R_{x}$ is the pullback via the automorphism $X \rightarrow X, z \mapsto x \cdot z$, and $T_{\mathcal{E}}$ denotes the Fourier-Mukai transform with kernel

$$
\operatorname{Cone}\left(\mathcal{E}^{\vee} \otimes \mathcal{E} \rightarrow \Delta_{*} \mathcal{O}_{X}\right), \quad \mathcal{E} \in \mathcal{D}^{\mathrm{b}}(X) .
$$

Now notice that there is the obvious homomorphism

$$
\gamma_{1}: X \times X \rightarrow \operatorname{Auteq}\left(\mathcal{D}^{\mathrm{b}}(X)\right) / \mathbb{Z}, \quad(x, y) \mapsto L_{x} R_{y} .
$$

More interestingly, using the known relations between the functors (13) (see [ST], Section 3d), one can easily check that $g_{1} \mapsto T_{\mathcal{O}_{x_{0}}}$ and $g_{2} \mapsto T_{\mathcal{O}}$ extend to a welldefined homomorphism

$$
\gamma_{2}: \mathrm{SL}_{2}(\mathbb{Z}) \rightarrow \operatorname{Auteq}\left(\mathcal{D}^{\mathrm{b}}(X)\right) / \mathbb{Z} .
$$

Another direct calculation shows that the maps (14) and (15) agree with each other extending to the semidirect product $(X \times X) \rtimes \mathrm{SL}_{2}(\mathbb{Z})$. By Theorem 3 , this defines the desired isomorphism (12). It would be very interesting to find a conceptual explanation for this isomorphism (cf. [ST], Remark 1.5). The results of [BaEG] as well as recent papers [SV] and [Po] suggest that the existence of $\gamma$ may not be a mere coincidence.

\section{Morita classification}

We begin by classifying the algebras $A_{q}$ up to Morita equivalence within the family $\left\{A_{q}\right\}$ and then consider the general case.

Theorem 4. Under the assumption (1), the algebras $A_{q}$ and $A_{q^{\prime}}$ are Morita equivalent if and only if they are isomorphic, i. e., if and only if $q^{\prime}=q$ or $q^{\prime}=q^{-1}$.

Proof. The fact that $A_{q} \cong A_{q^{\prime}} \Longleftrightarrow q^{\prime}=q^{ \pm 1}$ follows easily from the defining relations of $A_{q}$ and $A_{q^{\prime}}$ (see [J], Theorem 1.3). Now assume that $A_{q^{\prime}}$ and $A_{q}$ are Morita equivalent. Then $A_{q^{\prime}} \cong \operatorname{End}_{A_{q}}(M)$ for some f. g. projective right module $M$. Since $A_{q^{\prime}}$ is a domain, $M$ is isomorphic to a right ideal in $A_{q}$ (see, e.g., [BEG], Lemma 3). Since $\operatorname{End}_{A_{q}}(M) \cong A_{q^{\prime}}, \operatorname{Aut}_{A}(M) \not \mathbb{C}^{*}$. By Theorem 2, $M \cong A_{q}$. Therefore, $A_{q^{\prime}} \cong A_{q}$, which implies that $q^{\prime}=q^{ \pm 1}$. 
Remark. Theorem 4 was first proven in [RS] by a different method. If $q=e^{2 \pi i \theta}$ and $q^{\prime}=e^{2 \pi i \theta^{\prime}}$, with $\theta, \theta^{\prime} \in \mathbb{R} \backslash \mathbb{Q}$, then Theorem 4 says that $A_{\theta}$ and $A_{\theta^{\prime}}$ are equivalent if and only if $\theta^{\prime} \pm \theta \in \mathbb{Z}$. This result should be compared with a well-known Morita classification of smooth noncommutative tori $\mathscr{A}_{\theta}$ (see [R1]): in that case, $\mathscr{A}_{\theta}$ and $\mathcal{A}_{\theta^{\prime}}$ are (strongly) Morita equivalent if and only if $\theta$ and $\theta^{\prime}$ are in the same orbit of $\mathrm{GL}_{2}(\mathbb{Z})$ acting on $\mathbb{R} \backslash \mathbb{Q}$ by fractional linear transformations. Thus, unlike in the smooth case, there are no interesting Morita equivalences between the algebras $A_{q}$ for different values of $q$. However, in the Morita class of each $A_{q}$ there are many non-isomorphic algebras corresponding to different orbits of $\operatorname{Pic}\left(A_{q}\right)$ in $\mathcal{R}$. We will classify these algebras below, using Theorem 1.

We now describe an action of $\operatorname{Pic}\left(A_{q}\right)$ on the (reduced) Calogero-Moser spaces $\bar{\varphi}_{n}^{q}$. In Section 5, we will show that this action comes from a natural action of the braid group $B_{2}$ on the double affine Hecke algebra $\mathbb{H}_{1, \tau}\left(S_{n}\right)$ constructed by Cherednik in [C2]. We begin by defining an action of $\operatorname{Aut}\left(A_{q}\right)$ on $\bigodot_{n}^{q}$. With the identification of Lemma 3, it suffices to construct two compatible group homomorphisms

$$
f_{1}:\left(\mathbb{C}^{*}\right)^{2} \rightarrow \operatorname{Aut}\left(\varphi_{n}^{q}\right) \text { and } f_{2}: \operatorname{SL}_{2}(\mathbb{Z}) \rightarrow \operatorname{Aut}\left(\zeta_{n}^{q}\right),
$$

where $\operatorname{Aut}\left(\mathcal{C}_{n}^{q}\right)$ denotes the group of regular (algebraic) automorphisms of $\mathcal{C}_{n}^{q}$. First, we let

$$
f_{1}(\alpha, \beta):(X, Y) \mapsto\left(\alpha^{-1} X, \beta^{-1} Y\right), \quad(\alpha, \beta) \in\left(\mathbb{C}^{*}\right)^{2} .
$$

Next, to define the second homomorphism we will use the presentation (10): on the braid generators, we define $f_{2}$ by

$$
g_{1}:(X, Y) \mapsto\left(Y^{-1} X, Y\right), \quad g_{2}:(X, Y) \mapsto(X, Y X) .
$$

A direct calculation then shows that $f_{2}\left(g_{1} g_{2} g_{1}\right)=f_{2}\left(g_{2} g_{1} g_{2}\right)$ and $f_{2}\left(g_{1} g_{2}\right)^{6}=$ 1 in $\operatorname{Aut}\left(\mathcal{C}_{n}^{q}\right)$. Hence, this assignment extends to a well-defined homomorphism $f_{2}: \operatorname{SL}_{2}(\mathbb{Z}) \rightarrow \operatorname{Aut}\left(\boldsymbol{C}_{n}^{q}\right)$. It is also easy to check that $f_{1}$ and $f_{2}$ are compatible in the sense that

$$
f:=\left(f_{1}, f_{2}\right):\left(\mathbb{C}^{*}\right)^{2} \rtimes \operatorname{SL}_{2}(\mathbb{Z}) \rightarrow \operatorname{Aut}\left(\mathcal{C}_{n}^{q}\right) .
$$

is a group homomorphism. Now, with the identification of Theorem 3 , we see that $f$ induces

$$
\bar{f}: \operatorname{Pic}\left(A_{q}\right) \rightarrow \operatorname{Aut}\left(\overline{\mathcal{C}}_{n}^{q}\right),
$$

which defines an action of $\operatorname{Pic}\left(A_{q}\right)$ on each of the spaces $\bar{\varphi}_{n}^{q}$ and hence on their disjoint union $\bar{\complement}^{q}$. On the other hand, $\operatorname{Pic}\left(A_{q}\right)$ acts naturally on the space of ideal classes $\mathcal{R}^{q}=\mathcal{R}\left(A_{q}\right)$. With these actions, we have

Proposition 2. The map $\omega: \bar{e}^{q} \rightarrow \mathcal{R}^{q}$ of Theorem 1 is equivariant under $\operatorname{Pic}\left(A_{q}\right)$.

We will prove Proposition 2 in Section 5. Its meaning becomes clear from the following theorem, which gives a geometric classification of algebras Morita equivalent to $A_{q}$. 
Theorem 5. There is a natural bijection between the orbits of $\operatorname{Pic}\left(A_{q}\right)$ in $\bar{\complement}^{q}$ and the isomorphism classes of domains Morita equivalent to $A_{q}$.

Proof. The map $\omega: \bar{\complement}^{q} \rightarrow \mathcal{R}^{q}$ assigns to a point in $\bar{\ell}^{q}$ an isomorphism class $[M]$ of ideals in $A_{q}$. Choosing a representative $M$ in such a class and taking its endomorphism ring $\operatorname{End}_{A_{q}}(M)$ yields a domain Morita equivalent to $A_{q}$. Now a well-known result in ring theory (see, e.g, [F], Theorem 1) says that the $\operatorname{rings} \operatorname{End}_{A_{q}}(M)$ and $\operatorname{End}_{A_{q}}\left(M^{\prime}\right)$ are isomorphic iff the corresponding classes $[M]$ and $\left[M^{\prime}\right]$ are in the same orbit of $\operatorname{Pic}\left(A_{q}\right)$ in $\mathcal{R}_{q}$. Since, by the Morita Theorem, every domain equivalent to $A_{q}$ is of the form $\operatorname{End}_{A_{q}}(M)$, Theorem 5 follows immediately from Theorem 1 and Proposition 2.

Remark. If $D$ is an algebra Morita equivalent to $A_{q}$, which is not a domain, then $D \cong \mathrm{M}_{r}\left(A_{q}\right)$ for some $r \geq 2$. This follows from the fact that all projective modules over $A_{q}$ of rank $r \geq 2$ are free.

In general, the domains Morita equivalent to $A_{q}$ seem to have a complicated structure: they are not easy to describe in terms of generators and relations, like $A_{q}$. However, their automorphism groups can be described geometrically, in terms of the action of $\operatorname{Pic}\left(A_{q}\right)$ on $\bar{\ell}^{q}$. Precisely, we have the following consequence of Theorem 1 and Theorem 2.

Proposition 3. Let $M$ be a noncyclic right ideal of $A_{q}$, and let $E=\operatorname{End}_{A_{q}}(M)$ denote its endomorphism algebra. Then $\operatorname{Aut}_{\mathbb{C}}(E)$ is naturally isomorphic to the isotropy group of the point $\omega^{-1}[M]$, corresponding to the class of $M$ under the Calogero-Moser bijection, for the action of $\operatorname{Pic}\left(A_{q}\right)$ on $\bar{\complement}^{q}$ defined by (16).

Proof. Put $A:=A_{q}$. Then $M$ is naturally a $E$ - $A$-bimodule. Let $M^{*}:=\operatorname{Hom}_{A}(M, A)$ be its dual which is an $A-E$-bimodule. By the Morita Theorem, we have $M^{*} \otimes_{E} M \cong$ $A$ as $A$-bimodules and $M \otimes_{A} M^{*} \cong E$ as $E$-bimodules. Now consider the canonical sequence of groups

$$
1 \rightarrow \operatorname{Inn}(E) \rightarrow \operatorname{Aut}(E) \stackrel{\Omega}{\longrightarrow} \operatorname{Pic}(E) \stackrel{\Psi}{\longrightarrow} \operatorname{Pic}(A),
$$

where the last map is an isomorphism of groups given by $P \mapsto M^{*} \otimes_{E} P \otimes_{E} M$. The image of $\Omega$ consists of those invertible $E$-bimodules that are cyclic as right $E$-modules. Given such an $E$-bimodule $L$, we have

$$
M \otimes_{A} M^{*} \otimes_{E} L \otimes_{E} M \cong E \otimes_{E} L \otimes_{E} M \cong L \otimes_{E} M \cong M
$$

as right $A$-modules. Hence, $\Psi(L)=M^{*} \otimes_{E} L \otimes_{E} M$ is in the stabilizer of [M] under the action of $\operatorname{Pic}(A)$ on $\mathcal{R}^{q}$. Conversely, if $P$ is an invertible $A$-bimodule such that $M \otimes_{A} P \cong M$, then

$$
M \otimes_{A} P \otimes_{A} M^{*} \cong M \otimes_{A} M^{*} \cong E
$$


as right $E$-modules. Thus, $\Psi^{-1}(P) \cong M \otimes_{A} P \otimes_{A} M^{*}$ is in the image of $\Omega$. It follows now from Theorem 1 that $\operatorname{Im}(\Omega)$ is isomorphic to the stabilizer of $\omega^{-1}[M]$ under the $\operatorname{Pic}(A)$-action on $\bar{e}^{q}$. On the other hand, by Theorem 2, $\operatorname{Inn}(E)$ is trivial. Hence $\operatorname{Aut}(E) \cong \operatorname{Im}(\Omega)$. This finishes the proof of the proposition.

\section{Double affine Hecke algebras and the Calogero-Moser correspondence}

In this section, we describe a relation between $A_{q}$ and the double affine Hecke algebras $H_{q, n}:=\mathbb{U}_{1, q^{-1 / 2}}\left(S_{n}\right)$. Our construction generalizes (and simplifies) the results of [BCE], where a similar relation between $A_{1}$ and the rational Cherednik algebra has been studied. A key role in this construction is played by a multiplicative version of the deformed preprojective algebra of a quiver introduced in [CBS]. We draw the reader's attention to the fact that we use a more general form of these algebras in which weights are assigned not only to the vertices but also to the edges of the quiver. To simplify exposition we omit most routine calculations, especially those ones parallel to $[\mathrm{BCE}]$. However, we will give some details in the proof of Proposition 2, since the idea of this proof has not been used in the case of the Weyl algebra.

5.1. DAHA and Calogero-Moser spaces. We recall the presentation of the double affine Hecke algebra $H_{q, n}$ (see [C2]).

Generators:

$$
X_{1}^{ \pm 1}, X_{2}^{ \pm 1}, \ldots, X_{n}^{ \pm 1} ; \quad T_{1}, T_{2}, \ldots, T_{n-1} ; \quad \pi .
$$

Relations:

$$
\begin{aligned}
& X_{i} X_{j}=X_{j} X_{i}, \quad T_{i} X_{i} T_{i}=X_{i+1}, \quad \pi X_{i}=X_{i+1} \pi \quad(1 \leq i \leq n-1), \\
& T_{i} T_{i+1} T_{i}=T_{i+1} T_{i} T_{i+1}, \quad \pi T_{i}=T_{i+1} \pi \quad(1 \leq i \leq n-2), \\
& T_{i} X_{j}=X_{j} T_{i} \quad(j-i \neq 0,1), \quad\left[T_{i}, T_{j}\right]=0 \quad(|i-j|>1), \\
& \pi X_{n}=X_{1} \pi, \quad \pi^{n} T_{i}=T_{i} \pi^{n} \quad(1 \leq i \leq n-1), \\
& \left(T_{i}-\tau\right)\left(T_{i}+\tau^{-1}\right)=0, \quad \tau:=q^{-1 / 2} .
\end{aligned}
$$

Further, following [C2], we introduce $n$ pairwise commuting elements in $H_{q, n}$ :

$$
Y_{i}:=T_{i} T_{i+1} \ldots T_{n-1} \pi^{-1} T_{1}^{-1} \ldots T_{i-1}^{-1}, \quad i=1,2, \ldots, n,
$$

satisfying the relations

$$
T_{i} Y_{i+1} T_{i}=Y_{i} \quad \text { and } \quad T_{i} Y_{j}=Y_{j} T_{i}, \quad j-i \neq 0,1 .
$$

The algebra $H_{q, n}$ contains a copy of the finite Hecke algebra $\mathscr{H}_{\tau}\left(S_{n}\right)$ of the group $S_{n}$, which, in turn, contains the idempotents

$$
\varepsilon:=\frac{\sum_{w \in S_{n}} \tau^{l(w)} T_{w}}{\sum_{w \in S_{n}} \tau^{2 l(w)}} \quad \text { and } \quad \varepsilon^{\prime}:=\frac{\sum_{w \in S_{n-1}} \tau^{l(w)} T_{w}}{\sum_{w \in S_{n-1}} \tau^{2 l(w)}} .
$$


In formula (17), $S_{n-1}$ is regarded as the subgroup of $S_{n}$ consisting of permutations that fix the first element in $\{1,2, \ldots, n\}$; for $w \in S_{n}$, the element $T_{w}$ denotes the product $T_{i_{1}} \ldots T_{i_{l(w)}}$, where $w=s_{i_{1}} \ldots s_{i_{l(w)}}$ is a reduced expression of $w$ with $s_{i}:=(i, i+1) \in S_{n}$.

The following proposition is analogous to [EG], Theorem 1.23 and Theorem 1.24, in the case of the rational Cherednik algebra.

Proposition 4 ([O], Theorem 5.1 and Theorem 6.1). (1) The algebra $\varepsilon H_{q, n} \varepsilon$ is Morita equivalent to $H_{q, n}$, the equivalence $\operatorname{Mod}\left(H_{q, n}\right) \rightarrow \operatorname{Mod}\left(\varepsilon H_{q, n} \varepsilon\right)$ being the canonical functor $\varepsilon: M \mapsto \varepsilon M$.

(2) $\varepsilon H_{q, n} \varepsilon$ is a commutative algebra isomorphic to the coordinate ring of $\mathcal{C}_{n}^{q}$.

Next we introduce a multiplicative version of the deformed preprojective algebra of a quiver $Q$, due to Crawley-Boevey and Shaw [CBS] (see also [vdB]). Our definition is slightly more general than that of [CBS] as we assign weights to both the vertices and the arrows of $Q$ (see Remark below).

5.2. The (generalized) multiplicative preprojective algebra. Let $Q$ be a quiver with vertex set $I$. Let $\bar{Q}$ be the double of $Q$ obtained by adjoining a reverse arrow $a^{*}$ to each arrow $a \in Q$. As in [CBS], we extend $a \mapsto a^{*}$ to an involution on $\bar{Q}$ by letting $\left(a^{*}\right)^{*}=a$, and define the function $\epsilon: \bar{Q} \rightarrow\{ \pm 1\}$ by $\epsilon(a)=1$ if $a \in Q$ and $\epsilon(a)=-1$ if $a^{*} \in Q$. Next we choose two sets of parameters (weights): $\left\{q_{v}\right\}_{v \in I}$ and $\left\{\hbar_{a}\right\}_{a \in \bar{Q}}$ with the assumption that $\hbar_{a^{*}}=\hbar_{a}$ for all $a \in Q$. The multiplicative preprojective algebra $\Lambda^{q, \hbar}(Q)$ is now defined by the algebra homomorphism $\mathbb{C} \bar{Q} \rightarrow$ $\Lambda^{q, \hbar}(Q)$, which is universal among all algebra homomorphisms $\mathbb{C} \bar{Q} \rightarrow R$ satisfying the properties

$$
\begin{aligned}
& a a^{*}+\hbar_{a} \text { is a unit in } R \text { for all } a \in \bar{Q}, \\
& \prod_{a \in \bar{Q}}\left(a a^{*}+\hbar_{a}\right)^{\epsilon(a)}=\sum_{v \in I} q_{v} e_{v} \quad \text { in } R .
\end{aligned}
$$

Remark. The original definition of multiplicative preprojective algebras (see [CBS], Definition 1.2) corresponds to the choice $\hbar_{a}=1$ for all $a \in \bar{Q}$. To see why we need an extension of this definition consider a quiver $Q$ which consists of a single vertex $v$ and a single loop $a$. Choosing then $q_{v}=q$ and $\hbar_{a}=0$, we get the algebra $\Lambda^{q, \hbar}(Q)$ isomorphic to $A_{q}$ (with $a \leftrightarrow x$ and $a^{*} \leftrightarrow y$ ). On the other hand, Example 1.3 in [CBS] shows that if $q_{v}=q$ and $\hbar_{a}=1$, then $\Lambda^{q, \hbar}(Q) \cong$ $\mathbb{C}\left\langle x, y,(1+x y)^{-1}\right\rangle /(x y-q y x-1)$, which is a different quantized version of the first Weyl algebra, not isomorphic to $A_{q}$ (see, e.g., [AD2]).

Now, as in [BCE], Sect. 2.2, we consider the framed one-loop quiver $Q=Q_{\infty}$ with two vertices, $I=\{0, \infty\}$, and two arrows $i: 0 \rightarrow \infty$ and $X: 0 \rightarrow 0$. Write $j:=i^{*}$ and $Y:=X^{*}$ for the reverse arrows in $\bar{Q}$. For the vertex and arrow weights, we take $\left(q_{0}, q_{\infty}\right)=\left(q, q^{-n}\right)$ and $\left(\hbar_{X}, \hbar_{i}\right)=(0,1)$, respectively. The 
corresponding algebra $\Lambda:=\Lambda^{q, \hbar}(Q)$ can then be identified with the quotient of $\mathbb{C} \bar{Q}\langle U, V\rangle:=\mathbb{C} \bar{Q} * \mathbb{C}\langle U, V\rangle$ modulo the relations

$$
\begin{gathered}
U=e U e, \quad V=e V e, \quad X U=U X=e, \quad Y V=V Y=e, \\
X Y-q Y X-q Y X i j=0, \quad j i=\left(q^{-n}-1\right) e_{\infty},
\end{gathered}
$$

where $e$ and $e_{\infty}$ are the idempotents corresponding to the vertices 0 and $\infty$, respectively. The following lemma clarifies the relation between this algebra and $A_{q}$ : it is a "multiplicative" analogue of [BCE], Lemma 3.

Lemma 4. $A_{q}$ is isomorphic to the quotient of $\Lambda$ by the ideal generated by $e_{\infty}$.

In fact, the required isomorphism is induced by $\mathbb{C}\left\langle x^{ \pm 1}, y^{ \pm 1}\right\rangle \rightarrow \Lambda /\left\langle e_{\infty}\right\rangle, x \mapsto$ $X, y \mapsto Y$.

Next, we explain the relation between $\Lambda$ and the Cherednik algebra $H:=H_{q, n}$. To this end, we consider the left projective $H$-module $P:=H \varepsilon^{\prime} \oplus H \varepsilon$, where $\varepsilon$ and $\varepsilon^{\prime}$ are the idempotents defined in (17). The endomorphism ring of $P$ can be identified with a matrix algebra:

$$
\operatorname{End}_{H}(P)=\left(\begin{array}{cc}
\varepsilon^{\prime} H \varepsilon^{\prime} & \varepsilon^{\prime} H \varepsilon \\
\varepsilon H \varepsilon^{\prime} & \varepsilon H \varepsilon
\end{array}\right)
$$

Using this identification, we can define an algebra map $\Theta: \mathbb{C} \bar{Q}\langle U, V\rangle \rightarrow \operatorname{End}_{H}(P)^{\circ}$ by

$$
X \mapsto\left(\begin{array}{cc}
X_{1} \varepsilon^{\prime} & 0 \\
0 & 0
\end{array}\right), \quad Y \mapsto\left(\begin{array}{cc}
Y_{1} \varepsilon^{\prime} & 0 \\
0 & 0
\end{array}\right), \quad i \mapsto\left(\begin{array}{cc}
0 & 0 \\
\varepsilon & 0
\end{array}\right), \quad j \mapsto\left(\begin{array}{cc}
0 & \varepsilon \\
0 & 0
\end{array}\right)\left(q^{-n}-1\right) .
$$

The following proposition shows that $\Theta$ is a multiplicative analogue of the map $\Theta^{\text {quiver }}$ constructed in [EGGO] (see loc. cit., (1.6.3)).

Proposition 5. The map $\Theta$ induces an algebra homomorphism $\Lambda \rightarrow \operatorname{End}_{H}(P)^{\circ}$.

Proof. The key relation to verify is $\Theta(U V X Y-q e-q v w)=0$; all the other relations are immediate. First, using the fact that $\varepsilon \varepsilon^{\prime}=\varepsilon^{\prime} \varepsilon=\varepsilon$, we find

$$
\Theta(U V X Y-q e-q v w)=\left(\begin{array}{cc}
Y_{1} X_{1} Y_{1}^{-1} X_{1}^{-1} \varepsilon^{\prime}-q \varepsilon^{\prime}+q\left(1-q^{-n}\right) \varepsilon & 0 \\
0 & 0
\end{array}\right) .
$$

By [O], Lemma 4.1, $X_{1} Y_{1} X_{1}^{-1} Y_{1}^{-1}=T_{1}^{-1} T_{2}^{-1} \ldots T_{n-1}^{-2} T_{n-2}^{-1} \ldots T_{1}^{-1}$ in $H$. Hence $X_{1} Y_{1} X_{1}^{-1} Y_{1}^{-1}$ is an element of the finite Hecke algebra $\mathscr{H}_{\tau}\left(S_{n}\right) \subset H$. Now consider $\mathscr{H}_{\tau}\left(S_{n}\right)$ as a right $\mathscr{H}_{\tau}\left(S_{n-1}\right)$-module. Its subspace of invariants $V:=\mathscr{H}_{\tau}\left(S_{n}\right) \varepsilon^{\prime} \subset$ $\mathscr{H}_{\tau}\left(S_{n}\right)$ is then a left $\mathscr{H}_{\tau}\left(S_{n}\right)$-module. As shown in [O], Section 4.2, this last module admits a direct decomposition $V=\mathscr{H}_{\tau}\left(S_{n}\right) \varepsilon \oplus W$, where $W$ has dimension $n-1$. Moreover, the left multiplication by $Y_{1} X_{1} Y_{1}^{-1} X_{1}^{-1}$ acts on $\mathscr{H}_{\tau}\left(S_{n}\right) \varepsilon$ as a scalar operator with eigenvalue $q^{1-n}$ and on $W$ with eigenvalue $q$. The operator $q+q\left(q^{-n}-1\right) \varepsilon$ obviously acts on $V$ in the same way, so $Y_{1} X_{1} Y_{1}^{-1} X_{1}^{-1} \varepsilon^{\prime}-q \varepsilon^{\prime}+q\left(1-q^{-n}\right) \varepsilon=0$, which proves the desired identity. 
5.3. The Calogero-Moser functor. One way to interpret Proposition 5 is to say that $P$ is an $H$ - $\Lambda$-bimodule, with right $\Lambda$-module structure defined by $\Theta$. In combination with Lemma 4, this allows us to define the functor

$$
\mathrm{CM}_{n}: \mathcal{D}^{\mathrm{b}}(\operatorname{Mod} H) \rightarrow \mathcal{D}^{\mathrm{b}}(\operatorname{Mod} \Lambda) \rightarrow \mathcal{D}^{\mathrm{b}}\left(\operatorname{Mod} A_{q}\right), \quad V \mapsto\left(V \otimes_{H} P\right) \otimes_{\Lambda}^{L} A_{q} .
$$

Note that $P$ is a projective module on the left, so tensoring with $P$ over $H$ is an exact functor.

In view of Proposition 4, Theorem 1 of Section 2 is a consequence of the following result.

Theorem 6. (1) The functor $\mathrm{CM}_{n}$ transforms the simple $H$-modules (viewed as 0complexes in $\mathcal{D}^{\mathrm{b}}(\operatorname{Mod} H)$ ) to rank 1 projective $A_{q}$-modules (located in homological degree -1$)$.

(2) Two simple H-modules $V=\left(V ; X_{i}, T_{j}, \pi\right)$ and $V^{\prime}=\left(V^{\prime} ; X_{i}^{\prime}, T_{j}^{\prime}, \pi^{\prime}\right)$ correspond to isomorphic $A_{q}$-modules if and only if there is $(k, m) \in \mathbb{Z}^{2}$ such that

$$
X_{i}^{\prime}=q^{k} X_{i}, \quad T_{j}^{\prime}=T_{j}, \quad \pi^{\prime}=q^{m} \pi .
$$

(We call such $H$-modules equivalent.)

(3) For every rank 1 projective $A_{q}$-module, there is a unique $n \in \mathbb{N}$ and a simple module $V$ over $H_{q, n}$ such that $\mathrm{CM}_{n}(V) \cong M[1]$ in $\mathcal{D}^{\mathrm{b}}\left(\operatorname{Mod} A_{q}\right)$.

Thus the Calogero-Moser map $\omega$ of Theorem 1 is induced by the functors $\mathrm{CM}_{n}$ 'amalgamated' over all $n$. The proof of Theorem 6 is analogous to [BCE], Theorem 3: it is based on [BC], Theorem 3 , and the following key lemma.

To simplify the notation we set $R:=\mathbb{C}\left\langle x^{ \pm 1}, y^{ \pm 1}\right\rangle$ and denote by $\alpha$ the (surjective) algebra homomorphism $R \rightarrow e \Lambda e$ taking $x, x^{-1}, y, y^{-1}$ to $X, U, Y, V$, respectively. Using this homomorphism, we define the linear map

$$
\chi: R \rightarrow \varepsilon H \varepsilon, \quad r \mapsto \Theta(i \alpha(r) j) .
$$

Lemma 5. Regarding $P$ as a right $\Lambda$-module, we have:

(1) $\operatorname{Tor}_{k}^{\Lambda}\left(P, A_{q}\right)=0$ for all $k \neq 1$.

(2) For $k=1$, there is an isomorphism of right $A_{q}$-modules

$$
\operatorname{Tor}_{1}^{\Lambda}\left(P, A_{q}\right) \cong \operatorname{Ker}\left(\frac{H \varepsilon \otimes R}{H(\varepsilon \otimes r w-\chi(r) \otimes 1) R} \stackrel{\mu}{\longrightarrow} H \varepsilon^{\prime}\right),
$$

where $w=q^{-1}\left(x^{-1} y^{-1} x y\right)-1$.

The notation of Lemma 5 needs some explanation. The multiplication-action map $\mu$ in (18) is induced by $\mu(a \varepsilon \otimes r)=a \varepsilon \pi(r)$, where $a \varepsilon$ is viewed as an element in the direct summand of $P$ (note that $a \varepsilon=a \varepsilon \varepsilon^{\prime}$ ). The $A_{q}$-module structure on the right hand side of (18) descends then from the natural right $R$-module structure on $\operatorname{Ker} \mu$. 
Proof. (1) By Proposition 4(2), $\varepsilon H \varepsilon \cong \mathcal{O}\left(\bigodot_{n}^{q}\right)$. Write $\mathcal{O}:=\mathcal{O}\left(\bigodot_{n}^{q}\right)$. Then, for every maximal ideal $\mathfrak{m} \subset \mathcal{O},(\mathcal{O} / \mathfrak{m}) \otimes_{\mathcal{O}}(\varepsilon P)$ is a right $\Lambda$-module of dimension $n$ over $\mathbb{C}$. It follows that $(\mathcal{O} / \mathfrak{m}) \otimes_{\mathcal{O}}(\varepsilon P) \otimes_{\Lambda} A_{q}$ is a finite dimensional right $A_{q}$-module and hence zero. Since $\varepsilon P \otimes_{\Lambda} A_{q}$ is a coherent $\mathcal{O}$-module, this implies that $\varepsilon P \otimes_{\Lambda} A_{q}=0$ by Nakayama's Lemma. But then, by Morita equivalence of Proposition 4(1), $P \otimes_{\Lambda} A_{q}=0$. To see that the second and higher Tor's vanish we observe that the natural map $\Lambda e_{\infty} \Lambda \rightarrow \Lambda$ provides a left (and right) projective resolution of $A_{q}$, so $A_{q}$ has projective dimension 1 as a left $\Lambda$-module.

The proof of part (2) is similar to the proof of Theorem 1 in [BCE]. We leave it to the reader.

5.4. Equivariance. We now turn to the proof of Proposition 2. Recall that $\mathrm{SL}_{2}(\mathbb{Z})$ is a quotient of the braid group $B_{2}$. Let $\phi: B_{2} \rightarrow \mathrm{SL}_{2}(\mathbb{Z})$ denote the corresponding projection

$$
g_{1} \mapsto\left(\begin{array}{ll}
1 & 1 \\
0 & 1
\end{array}\right), \quad g_{2} \mapsto\left(\begin{array}{cc}
1 & 0 \\
-1 & 1
\end{array}\right) .
$$

The $\mathrm{SL}_{2}(\mathbb{Z})$-action on $\left(\mathbb{C}^{*}\right)^{2}$ defined by (9) induces (via $\phi$ ) a $B_{2}$-action on $\left(\mathbb{C}^{*}\right)^{2}$. We write $G:=\left(\mathbb{C}^{*}\right)^{2} \rtimes B_{2}$ for the corresponding semidirect product of groups.

Lemma 6 ([C2]). The following assignment extends to a well-defined group homomorphism $\Phi_{H}: G \rightarrow \operatorname{Aut}_{\mathbb{C}}\left(H_{q, n}\right)$ :

$$
\begin{aligned}
& (\alpha, \beta ; \text { id }) \mapsto v_{\alpha, \beta}:=\left(X_{i} \mapsto \alpha X_{i}, Y_{i} \mapsto \beta Y_{i}, T_{i} \mapsto T_{i}\right), \\
& \left(1,1 ; g_{1}\right) \mapsto \tau:=\left(X_{i} \mapsto Y_{i} X_{i}, Y_{i} \mapsto Y_{i}, T_{i} \mapsto T_{i}\right), \\
& \left(1,1 ; g_{2}\right) \mapsto \theta:=\left(X_{i} \mapsto X_{i}, Y_{i} \mapsto X_{i}^{-1} Y_{i}, T_{i} \mapsto T_{i}\right) .
\end{aligned}
$$

Proof. It is obvious that $v_{\alpha, \beta} \in \operatorname{Aut}_{\mathbb{C}}\left(H_{q, n}\right)$. The fact that $\theta$ and $\tau$ are also automorphisms, satisfying the braid relation $\theta \tau \theta=\tau \theta \tau$, is part of [C2], Theorem 4.3. (In [C2], $\theta^{-1}$ and $\tau$ are denoted by $\tau_{+}$and $\tau_{-}$, respectively). The following calculation now completes the proof:

$$
\begin{aligned}
\left(1,1 ; g_{1}\right)(\alpha, \beta ; \mathrm{id})\left(X_{i}, Y_{i}, T_{i}\right) & =\left(\alpha \beta Y_{i} X_{i}, \beta Y_{i}, T_{i}\right) \\
& =(\alpha \beta, \beta ; \mathrm{id})\left(1,1 ; g_{1}\right)\left(X_{i}, Y_{i}, T_{i}\right) \\
& =\left(g_{1}(\alpha, \beta) ; g_{1}\right)\left(X_{i}, Y_{i}, T_{i}\right), \\
\left(1,1 ; g_{2}\right)(\alpha, \beta ; \mathrm{id})\left(X_{i}, Y_{i}, T_{i}\right) & =\left(\alpha X_{i}, \alpha^{-1} \beta X_{i}^{-1} Y_{i}, T_{i}\right) \\
& =\left(\alpha, \alpha^{-1} \beta ; \mathrm{id}\right)\left(1,1 ; g_{2}\right)\left(X_{i}, Y_{i}, T_{i}\right) \\
& =\left(g_{2}(\alpha, \beta) ; g_{2}\right)\left(X_{i}, Y_{i}, T_{i}\right) .
\end{aligned}
$$

Note that any automorphism of $H$ in the image of $\Phi_{H}$ fixes the generators $T_{1}, \ldots$, $T_{n-1}$. It follows that the $G$-action on $H$ defined by $\Psi_{H}$ induces a $G$-action on the spherical algebra $\varepsilon H \varepsilon$ and the left $\varepsilon H \varepsilon$-module $\varepsilon P=\varepsilon H \varepsilon^{\prime} \oplus \varepsilon H \varepsilon$. In other words, 
we have group homomorphisms $\Phi_{\varepsilon H \varepsilon}: G \rightarrow \operatorname{Aut}_{\mathbb{C}}(\varepsilon H \varepsilon)$ and $\Phi_{P}: G \rightarrow \operatorname{Aut}_{\mathbb{C}}(\varepsilon P)$. In addition, we have

Lemma 7. The following assignment extends to a well-defined group homomorphism $\Phi_{\Lambda}: G \rightarrow \operatorname{Aut}_{\mathbb{C}}(\Lambda):$

$$
\begin{aligned}
& \left(1,1 ; g_{1}\right) \mapsto\left(X \mapsto X Y, Y \mapsto Y, j \mapsto Y^{-1} j, i \mapsto i Y\right), \\
& \left(1,1 ; g_{2}\right) \mapsto\left(X \mapsto X, Y \mapsto Y X^{-1}, j \mapsto X j, i \mapsto i X^{-1}\right), \\
& (\alpha, \beta ; \text { id }) \mapsto(X \mapsto \alpha X, Y \mapsto \beta Y, j \mapsto j, i \mapsto i) .
\end{aligned}
$$

Proof. A direct calculation similar to that of Lemma 6. We leave details to the reader.

Note that the above action of $G$ on $\Lambda$ preserves the idempotents $e$ and $e_{\infty}$ and hence restricts to the subalgebra $e \Lambda e \subset \Lambda$. By Proposition 5, $\varepsilon P$ is an $\varepsilon H \varepsilon$ - $\Lambda$-bimodule. The subspace $\varepsilon P e:=\varepsilon_{\varepsilon} P \otimes_{\Lambda} \Lambda e \subseteq \varepsilon P$ is preserved by any automorphism in Aut $\mathbb{C}(\varepsilon P)$, which is in the image of $\Phi_{P}$. As a result, we have an action of $G$ on $\varepsilon P e$. Now form the semidirect product $\left[\varepsilon H \varepsilon \otimes(e \Lambda e)^{\circ}\right] \rtimes G$, with $G$ acting diagonally on $\varepsilon H \varepsilon$ and $e \Lambda e$ as in Lemma 6 and Lemma 7.

Proposition 6. The action of $G$ on $\varepsilon P e$ defined above makes it a $\left[\varepsilon H \varepsilon \otimes(e \Lambda e)^{\circ}\right] \rtimes G$ module (equivalently, $\varepsilon P e$ is a $G$-equivariant $\varepsilon H \varepsilon$-e $\Lambda e$-bimodule).

Proof. We need to verify that, for all $h \in \varepsilon H \varepsilon, m \in \varepsilon P e, x \in e \Lambda e$ and $\sigma \in G$,

$$
(\sigma \cdot h)(\sigma . m)(\Theta(\sigma \cdot x))=\sigma \cdot(h m \Theta(x)) .
$$

Note that $\varepsilon P e$ may be identified with the direct summand $\varepsilon H \varepsilon^{\prime}$ of $\varepsilon P$. The $G$-action on $\varepsilon H \varepsilon^{\prime}$ as well as that on $\varepsilon H \varepsilon$ are obtained by restricting $\Phi_{H}$. It follows that $\sigma(h \cdot m)=(\sigma \cdot h)(\sigma \cdot m)$ for all $\sigma \in G, h \in \varepsilon H \varepsilon$ and $m \in \varepsilon P e$. Therefore, it suffices to verify (19) with $h=1$. For this, it suffices to show that $\Theta:(e \Lambda e)^{\circ} \rightarrow \varepsilon^{\prime} H \varepsilon^{\prime}$ is a $G$-module homomorphism. The following computation completes this verification (remember that we are working in the opposite algebra $\left.(e \Lambda e)^{\circ}\right)$.

$$
\begin{aligned}
& \Theta\left(\left(1,1 ; g_{1}\right)(X)\right)=\Theta(Y X)=Y_{1} X_{1} \varepsilon^{\prime}=\left(1,1 ; g_{1}\right) \Theta(X), \\
& \Theta\left(\left(1,1 ; g_{1}\right)(Y)\right)=\Theta(Y)=Y_{1} \varepsilon^{\prime}=\left(1,1 ; g_{1}\right) \Theta(Y), \\
& \Theta\left(\left(1,1 ; g_{2}\right)(X)\right)=\Theta(X)=X_{1} \varepsilon^{\prime}=\left(1,1 ; g_{2}\right) \Theta(X), \\
& \Theta\left(\left(1,1 ; g_{2}\right)(Y)\right)=\Theta\left(Y X^{-1}\right)=Y_{1} X_{1}^{-1} \varepsilon^{\prime}=\left(1,1 ; g_{2}\right) \Theta(Y), \\
& \Theta((\alpha, \beta ; \text { id })(X))=\Theta(\alpha X)=\alpha X_{1} \varepsilon^{\prime}=(\alpha, \beta ; \text { id }) \Theta(X), \\
& \Theta((\alpha, \beta ; \text { id })(Y))=\Theta(\beta Y)=\beta Y_{1} \varepsilon^{\prime}=(\alpha, \beta ; \text { id }) \Theta(Y) .
\end{aligned}
$$


Proof of Proposition 2. To simplify the notation, we set $\Gamma:=\varepsilon H \varepsilon \otimes(e \Lambda e)^{\circ}$ and $\Gamma_{A}:=\varepsilon H \varepsilon \otimes A_{q}^{\circ}$. Note that $G$ acts naturally on $A_{q}$. Further, the algebra homomorphism $e \Lambda e \rightarrow A_{q}$ given by Lemma 4 is $G$-equivariant. Hence, the induced homomorphism $\Gamma \rightarrow \Gamma_{A}$ is $G$-equivariant. Now, for any $G$-equivariant $\Gamma$-module $M$, we have an isomorphism $\Gamma_{A}$-modules

$$
M \otimes_{e \Lambda e} A_{q} \cong \Gamma_{A} \otimes_{\Gamma} M \cong\left(\Gamma_{A} \rtimes G\right) \otimes_{\Gamma \rtimes G} M .
$$

It follows that $M \otimes_{e \Lambda e}^{L} A_{q} \cong\left(\Gamma_{A} \rtimes G\right) \otimes_{\Gamma \rtimes G}^{L} M$ in the derived category of $\varepsilon H \varepsilon$ - $A_{q}$-bimodules. Hence $M \otimes_{e \Lambda e}^{L} A_{q}$ is a $G$-equivariant $\varepsilon H \varepsilon$ - $A_{q}$-bimodule. By Proposition 6, the $\varepsilon H \varepsilon-A_{q}$-bimodule $\tilde{P}:=\varepsilon P e \otimes^{L} A_{q}$ is $G$-equivariant. Identify $\varepsilon H \varepsilon \cong \mathcal{O}\left(\bigodot_{n}^{q}\right)$ as in Proposition 4, and let $\mu: \mathcal{O} \otimes \tilde{P} \otimes A_{q} \rightarrow \tilde{P}$ denote the structure map of the bimodule $\tilde{P}$ with this identification. Now, for any $\sigma \in G$ and for any maximal ideal $\mathfrak{m} \subset \mathcal{O}$, the commutative diagram

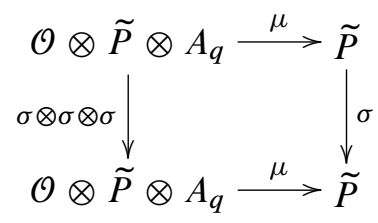

induces

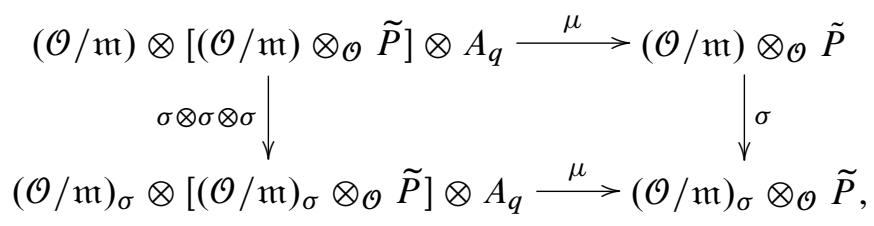

where $(\mathcal{O} / \mathfrak{m})_{\sigma}$ denotes the twisting by $\sigma$ of the $\mathcal{O}$-module $\mathcal{O} / \mathfrak{m} \cong \mathbb{C}$. It follows that the map $\omega_{n}: \mathcal{C}_{n}^{q} \rightarrow \mathcal{R}\left(A_{q}\right)$ is $G$-equivariant. Proposition 2 follows once we note that, by Theorem $3, \operatorname{Pic}\left(A_{q}\right) \cong G / \mathbb{Z}^{2}$, and the $G$-action on $\ell_{n}^{q}$ descends to the $\operatorname{Pic}\left(A_{q}\right)$-action on $\bar{\ell}_{n}^{q}$.

\section{Appendix: Comparison with noncommutative tori in the smooth case}

In the following table, we compare the properties of algebraic and smooth noncommutative tori. In the algebraic case, most ring-theoretic and homological properties follow from the fact that $A_{q}$ is a simple hereditary domain (see [J]); the classification of projectives and Morita classification are results of this paper, and the computation of Hochschild and cyclic homology can be found in [Wa]. In the smooth case, the description of projective modules and Morita classification can be found in [R2], the Picard group of $\mathcal{A}_{q}$ is computed in [K1], and results on Hochschild, cyclic homology, and homological dimension follow from [Co]. 
Algebraic vs smooth noncommutative tori

\begin{tabular}{|c|c|c|}
\hline Properties & $\begin{array}{l}\text { Algebraic torus } \\
A_{q}\left(q^{n} \neq 1, n \in \mathbb{Z}\right)\end{array}$ & $\begin{array}{l}\text { Smooth torus } \\
\mathscr{A}_{\theta}\left(q=e^{2 \pi i \theta}, \theta \in \mathbb{R} \backslash \mathbb{Q}\right)\end{array}$ \\
\hline Cancelation & $\begin{array}{l}\text { Any f.g. projective module of rank } \\
\geq 2 \text { is free. }\end{array}$ & $\begin{array}{l}\text { Stably isomorphic f.g. projective } \\
\text { modules are isomorphic. }\end{array}$ \\
\hline $\begin{array}{l}\text { Projective } \\
\text { modules }\end{array}$ & $\begin{array}{l}\text { Any f.g. projective module is } \\
\text { either free or isomorphic to a right } \\
\text { ideal. }\end{array}$ & $\begin{array}{l}\text { Any f.g. projective module is } \\
\text { isomorphic to a standard module } \\
\text { introduced by Connes. }\end{array}$ \\
\hline $\begin{array}{l}\text { Grothendieck } \\
\text { group }\end{array}$ & $\mathbb{Z}$ & $\mathbb{Z}^{2}$ \\
\hline $\begin{array}{l}\text { Isomorphism } \\
\text { classes }\end{array}$ & $\begin{array}{l}A_{q} \text { is isomorphic to } A_{q^{\prime}} \text { if and } \\
\text { only if } q^{\prime}=q^{ \pm 1} \text {. }\end{array}$ & $\begin{array}{l}\mathcal{A}_{\theta} \text { is isomorphic to } \mathcal{A}_{\theta^{\prime}} \text { if and } \\
\text { only if } \theta \pm \theta^{\prime} \in \mathbb{Z} .\end{array}$ \\
\hline Morita classes & $\begin{array}{l}A_{q} \text { is Morita equivalent to } A_{q^{\prime}} \text { if } \\
\text { and only if } q^{\prime}=q^{ \pm 1} \text {. A unital } \\
\text { algebra is Morita equivalent to } A_{q} \\
\text { if and only if } A \text { is isomorphic to } \\
M_{n}\left(A_{q}\right) \text { for some } n \geq 2 \text { or } \\
\operatorname{End}_{A_{q}}(M) \text { for some right ideal } \\
M \text { of } A_{q} .\end{array}$ & $\begin{array}{l}\text { A unital } C^{*} \text {-algebra is Morita } \\
\text { equivalent to } \mathscr{A}_{\theta} \text { if and only if it } \\
\text { is isomorphic } \mathbb{M}_{n}\left(\mathscr{A}_{\theta^{\prime}}\right) \text { for } \\
\text { some } n \geq 2 \text {, and } \theta^{\prime}=\frac{a \theta+b}{c \theta+d}, \\
\text { where }\left(\begin{array}{ll}a & b \\
c & d\end{array}\right) \in \operatorname{GL}(2, \mathbb{Z}) .\end{array}$ \\
\hline $\begin{array}{l}\text { Outer } \\
\text { automorphism } \\
\text { group }\end{array}$ & $\left(\mathbb{C}^{*} / \mathbb{Z}\right)^{2} \rtimes \operatorname{SL}(2, \mathbb{Z})$ & $\left(\mathbb{C}^{*} / \mathbb{Z}\right)^{2} \rtimes \operatorname{SL}(2, \mathbb{Z})$ \\
\hline Picard group & $\left(\mathbb{C}^{*} / \mathbb{Z}\right)^{2} \rtimes \operatorname{SL}(2, \mathbb{Z})$ & $\begin{array}{l}\text { If } \theta \text { is not quadratic, } \operatorname{Pic}\left(\mathscr{A}_{\theta}\right) \text { is } \\
\text { isomorphic to } \operatorname{Out}\left(\mathcal{A}_{\theta}\right) \text {; if } \theta \text { is } \\
\text { quadratic, } \operatorname{Pic}\left(\mathscr{A}_{\theta}\right) \text { is } \\
\text { isomorphic to }\left(\operatorname{Out} \mathscr{A}_{\theta}\right) \rtimes \mathbb{Z} \text {. }\end{array}$ \\
\hline $\begin{array}{l}\text { Hochschild } \\
\text { homology }\end{array}$ & $\mathrm{HH}_{n}\left(A_{q}\right)= \begin{cases}\mathbb{C}, & n=0,2 \\
\mathbb{C}^{2}, & n=1 \\
0, & n>2\end{cases}$ & $\begin{array}{l}\text { If } \theta \text { satisfies a Diophantine } \\
\text { condition, } \\
\mathrm{HH}_{n}\left(\mathscr{A}_{\theta}\right)= \begin{cases}\mathbb{C}, & n=0,2, \\
\mathbb{C}^{2}, & n=1, \\
0, & n>2 .\end{cases} \\
\text { If } \theta \text { does not satisfy a } \\
\text { Diophantine condition, } \\
\mathrm{HH}_{0}\left(\mathscr{A}_{\theta}\right) \text { and } \mathrm{HH}_{1}\left(\mathscr{A}_{\theta}\right) \text { are } \\
\text { infinite-dimensional. }\end{array}$ \\
\hline $\begin{array}{l}\text { Cyclic } \\
\text { homology }\end{array}$ & $\mathrm{HP}_{\mathrm{odd}}\left(A_{q}\right)=\mathrm{HP}_{\mathrm{ev}}\left(A_{q}\right)=\mathbb{C}^{2}$ & $\mathrm{HP}_{\mathrm{odd}}\left(\mathcal{A}_{\theta}\right)=\mathrm{HP}_{\mathrm{ev}}\left(\mathcal{A}_{\theta}\right)=\mathbb{C}^{2}$ \\
\hline $\begin{array}{l}\text { Homological } \\
\text { dimension }\end{array}$ & 1 & 2 \\
\hline
\end{tabular}




\section{References}

[AD1] J. Alev and F. Dumas, Automorphismes de certains complétés du corps de Weyl quantique. Collect. Math. 46 (1995), 1-9. Zbl 0842.16022 MR 1366124

[AD2] J. Alev and F. Dumas, Rigidité des plongements des quotients primitifs minimaux de $U_{q}(\operatorname{sl}(2))$ dans l'algèbre quantique de Weyl-Hayashi. Nagoya Math. J. 143 (1996), 119-146. Zbl 0862.16019 MR 1413010

[BaEG] V. Baranovsky, S. Evens, and V. Ginzburg, Representations of quantum tori and $G$-bundles on elliptic curves. In The orbit method in geometry and physics (Marseille, 2000), Progr. Math. 213, Birkhäuser, Boston 2003, 29-48. Zbl 1161.17310 MR 1995373

[BC1] Yu. Berest and O. Chalykh, Ideals of quantum Weyl algebras. Unpublished notes, 2004.

[BC] Yu. Berest and O. Chalykh, $A_{\infty}$-modules and Calogero-Moser spaces. J. Reine Angew. Math. 607 (2007), 69-112. Zbl 1160.16005 MR 2338121

[BCE] Yu. Berest, O. Chalykh, and F. Eshmatov, Recollement of deformed preprojective algebras and the Calogero-Moser correspondence. Moscow Math. J. 8(2008), 21-37, 183. Zbl 1207.16034 MR 2422265

[BEG] Yu. Berest, P. Etingof, and V. Ginzburg, Morita equivalence of Cherednik algebras. J. Reine Angew. Math. 568 (2004), 81-98. Zbl 1067.16046 MR 2034924

[BW1] Yu. Berest and G. Wilson, Automorphisms and ideals of the Weyl algebra. Math. Ann. 318 (2000), 127-147. Zbl 0983.16021 MR 1785579

[BW2] Yu. Berest and G. Wilson, Ideal classes of the Weyl algebra and noncommutative projective geometry. Internat. Math. Res. Notices 2002 (2002), 1347-1396. Zbl 1055.16030 MR 1904791

[CH] R. C. Cannings and M. P. Holland, Etale covers, bimodules and differential operators. Math. Z. 216 (1994), 179-194. Zbl 0807.13011 MR 1278419

[CH1] R. C. Cannings and M. P. Holland, Rings of operators on modules over commutative rings and their right ideals. J. Algebra 186 (1996), 235-263. Zbl 0868.16022 MR 1418048

[CN] O. Chalykh and F. Nijhoff, Bispectral rings of difference operators. Uspekhi Mat. Nauk 54 (1999), no. 3, 173-174; English transl. Russian Math. Surveys 54 (1999), 644-645.

[C1] I. Cherednik, Double affine Hecke algebras, Knizhnik-Zamolodchikov equations, and Macdonald's operators. Internat. Math. Res. Notices 1992 (1992), 171-180. Zbl 0770.17004 MR 1185831

[C2] I. Cherednik, Macdonald's evaluation conjectures and difference Fourier transform. Invent. Math. 122 (1995), 119-145. Zbl 0854.22021 MR 1354956

[Co] A. Connes, Non-commutative differential geometry. Inst. Hautes Études Sci. Publ. Math. 62 (1985), 41-144. Zbl 0592.46056 MR 0823176

[CBS] W. Crawley-Boevey and P. Shaw, Multiplicative preprojective algebras, middle convolution and the Deligne-Simpson problem. Adv. Math. 201 (2006), 180-208. Zbl 1095.15014 MR 2204754 
[D] J. Dixmier, Sur les algèbres de Weyl. Bull. Soc. Math. France 96 (1968), 209-242. Zbl 0165.04901 MR 0242897

[EG] P. Etingof and V. Ginzburg, Symplectic reflection algebras, Calogero-Moser space, and deformed Harish-Chandra homomorphism. Invent. Math. 147 (2002), 243-348. Zbl 1061.16032 MR 1881922

[EGGO] P. Etingof, W. L. Gan, V. Ginzburg, and A. Oblomkov, Harish-Chandra homomorphisms and symplectic reflection algebras for wreath-products. Publ. Math. Inst. Hautes Études Sci. 105 (2007), 91-155. Zbl 1188.16010 MR 2354206

[FR] V. V. Fock and A. A. Rosly, Poisson structure on moduli of flat connections on Riemann surfaces and the $r$-matrix. In Moscow Seminar in mathematical physics, Amer. Math. Soc. Transl. Ser. (2) 191, Amer. Math. Soc., Providence, RI, 1999, 67-86. Zbl 0945.53050 MR 1730456

[F] A. Fröhlich, The Picard group of noncommutative rings, in particular of orders. Trans. Amer. Math. Soc. 180 (1973), 1-45. Zbl 0278.16016 MR 0318204

[FGL] C. Frohman, R. Gelca, and W. Lofaro, The A-polynomial from the noncommutative viewpoint. Trans. Amer. Math. Soc. 354 (2002), 735-747. Zbl 0980.57002 MR 1862565

[G] S. Gukov, Three-dimensional quantum gravity, Chern-Simons theory, and the Apolynomial. Comm. Math. Phys. 255(2005), 577-627. Zbl 1115.57009 MR 2134725

[J] V. A. Jategaonkar, A multiplicative analog of the Weyl algebra. Comm. Algebra 12 (1984), 1669-1688. Zbl 0581.16002 MR 743310

[KKO] A. Kapustin, A. Kuznetsov, and D. Orlov, Noncommutative instantons and twistor transform. Comm. Math. Phys. 221 (2001), 385-432. Zbl 0989.81127 MR 1845330

[K1] K. Kodaka, Picard groups of irrational rotation $C^{*}$-algebras. J. London Math. Soc. (2) 56 (1997), 179-188. Zbl 0892.46067 MR 1462834

[K2] K. Kodaka, $C^{*}$-algebras that are isomorphic after tensoring and full projections. Proc. Edinburgh Math. Soc. (2) 47 (2004), 659-668. Zbl 1075.46047 MR 2097266

[NB] K. de Naeghel and M. van den Bergh, Ideal classes of three-dimensional Sklyanin algebras. J. Algebra 276 (2004), 515-551. Zbl 1069.16033 MR 2058456

[NS] T. A. Nevins and J. T. Stafford, Sklyanin algebras and Hilbert schemes of points. Adv. Math. 210 (2007), 405-478. Zbl 1116.14003 MR 2303228

[O] A. Oblomkov, Double affine Hecke algebras and Calogero-Moser spaces. Represent. Theory 8 (2004), 243-266. Zbl 1078.20004 MR 2077482

[Or] D. O. Orlov, Derived categories of coherent sheaves on Abelian varieties and equivalences between them. Izv. Ross. Akad. Nauk Ser. Mat. 66 (2002), 131-158; English transl. Izv. Math. 66 (2002), 569-594. Zbl 1031.18007 MR 1921811

[Po] A. Polishchuk, Noncommutative two-tori with real multiplication as noncommutative projective varieties. J. Geom. Phys. 50 (2004), 162-187. Zbl 1085.14003 MR 2078224

[RS] L. Richard and A. Solotar, Isomorphisms between quantum generalized Weyl algebras. J. Algebra Appl. 5 (2006), 271-285. Zbl 1102.16025 MR 2235811 
[R1] M. A. Rieffel, $C^{*}$-algebras associated with irrational rotations. Pacific J. Math. 93 (1981), 415-429. Zbl 0499.46039 MR 623572

[R2] M. A. Rieffel, The cancellation theorem for projective modules over irrational rotation $C^{*}$-algebras. Proc. London Math. Soc. (3) 47 (1983), 285-302. Zbl 0541.46055 MR 703981

[R3] M. A. Rieffel, Non-commutative tori - a case study of noncommutative differentiable manifolds. In Geometric and topological invariants of elliptic operators (Brunswick, ME, 1988), Contemp. Math. 105, Amer. Math. Soc., Providence, RI, 1990, 191-211. Zbl 0713.46046 MR 1047281

[ST] P. Seidel and R. Thomas, Braid group actions on derived categories of coherent sheaves. Duke Math. J. 108 (2001), 37-108. Zbl 1092.14025 MR 1831820

[SV] Y. Soibelman and V. Vologodsky, Noncommutative compactifications and elliptic curves. Internat Math. Res. Notices 2003 (2003), 1549-1569. Zbl 1052.14004 MR 1976601

[St] J. T. Stafford, Endomorphisms of right ideals of the Weyl algebra. Trans. Amer. Math. Soc. 299 (1987), 623-639. Zbl 0615.16022 MR 869225

[vdB] M. Van den Bergh, Non-commutative quasi-Hamiltonian spaces. In Poisson geometry in mathematics and physics, Contemp. Math. 450, Amer. Math. Soc., Providence, RI, 2008, 273-299. Zbl 1236.58019 MR 2397630

[Wa] M. Wambst, Hochschild and cyclic homology of the quantum multiparametric torus. J. Pure Appl. Algebra 114 (1997), 321-329. Zbl 0881.18014 MR 1426492

[We] D. B. Webber, Ideals and modules of simple Noetherian hereditary rings. J. Algebra 16 (1970), 239-242. Zbl 0211.06201 MR 0265395

[W2] G. Wilson, Bispectral commutative ordinary differential operators. J. Reine Angew. Math. 442 (1993), 177-204. Zbl 0781.34051 MR 1234841

[W1] G. Wilson, Collisions of Calogero-Moser particles and an adelic Grassmannian. Invent. Math. 133 (1998), 1-41. Zbl 0906.35089 MR 1626461

Received October 19, 2010; revised February 15, 2011

Yu. Berest, Department of Mathematics, Cornell University, Ithaca, NY 14853-4201, U.S.A.

E-mail: berest@math.cornell.edu

A. Ramadoss, Departement Mathematik, ETH Zürich, 8092 Zürich, Switzerland

E-mail: ajay.ramadoss@math.ethz.ch

X. Tang, Department of Mathematics, Washington University, St. Louis, MO 63139, U.S.A.

E-mail: xtang@math.wustl.edu 